médecine/sciences $1986 ; 2: 220$

Mladie de

Creutzfeldt-Jakob et hormone

\section{de croissance}

Plusieurs articles très alarmistes ont paru voici quelques mois dans la presse anglo-saxonne [r], attirant l'attention sur le cas de patients ayant contracté une maladie de Creutzfeldt-Jakob, après traitement prolongé par hormone de croissance provenant d'hypophyses humaines; articles suivis d'un commentaire très documenté publié par $L a$ Recherche [2] fin 1985. Des informations récentes, plus optimistes, nous incitent à reprendre ce sujet.

La maladie de Creutzfeldt-Jakob est une dégénérescence subaiguë transmissible du système nerveux central: elle possède un équivalent animal, la tremblante (scrapie). C'est une affection de l'âge mûr, puisque sur les quelque $3000 \mathrm{cas}$ répertoriés, on n'en connaissait que 9 de moins de 30 ans, dont trois suspects d'origine iatrogène (par exemple après emploi stéréotaxique d'électrodes antérieurement implantées chez d'autres malades). Or, en l'espace d'un an, au moins quatre (et peut-être six) sujets de 20 à 34 ans sont morts de cette maladie; tous avaient subi un traitement prolongé par l'hormone de croissance humaine. Les chances qu'il n'y ait pas de relation entre le traitement et la maladie ont été évaluées à moins de I sur $10^{12}$.

Il apparaît certain que des lots d'hypophyse (qui comprennent selon les cas de 500 à 20000 glandes) sont contaminés par l'agent de la maladie de Creutzfeldt-Jakob, qui n'est donc pas inactivé par les traitements utilisés pendant la purification. Les problèmes que soulève cette constatation sont multiples.

Sur le plan épidémiologique deux hypothèses sont en présence: la maladie comporte habituellement une longue incubation. Les cas qui viennent de se déclarer concernent des patients qui ont reçu de l'hormone pendant des années. Représentent-ils le début d'une épidémie qui risque de faire tache d'huile au cours des prochaines années, ou bien constituent-ils l'ensemble, ou du moins l'essentiel, de l'épidémie? Rien à l'heure actuelle ne permet de trancher.

Le problème est donc désormais de savoir quelle attitude adopter à l'égard des milliers de sujets justiciables du traitement par l'hormone de croissance. Son utilisation a été suspendue aux USA et en GrandeBretagne, mais non en France. Les tests de présence du virus sont de deux types: l'un, très sensible, passe par son inoculation à des singes mais la réponse exige un très long délai (la période d'incubation étant d'au moins un an chez le chimpanzé et de deux ans chez le singe écureuil). L'autre test est biochimique: il existe une protéine de 27 kilodaltons, bien identifiée, caractéristique des tissus atteints; sa recherche donne des résultats immédiats mais elle est beaucoup moins sensible. Ces observations soulignent à nouveau les risques que fait courir aux malades l'usage de produits biologiques, du sang aux tissus et aux greffes. Elles renforcent le camp de ceux qui s'efforcent d'en remplacer le plus grand nombre possible. Dans le cas de l'hormone de croissance, c'est la production par génie génétique qui porte tous les espoirs mais celle-ci n'est pas encore parvenue à surmonter toutes les difficultés pratiques. Doit-on dès lors attendre passivement qu'elles le soient?

En réalité, les essais se sont multipliés pour débarrasser les préparations des virus qui pourraient les contaminer. L'agent pathogène est extrêmement résistant aux agents chimiques et à la chaleur, mais d'autres méthodes semblent efficaces. Des passages sur gel de dextrane et sur échangeurs d'ions ont paru éliminer le virus de la scrapie [3]. Très récemment [4], des auteurs japonais ont montré que ce type de virus traverse des filtres dont les pores sont de 100 nanomètres mais sont arrêtés par des pores de $25 \mathrm{~nm}$. Ils proposent donc une méthode de filtration sur des membranes ayant une taille des pores de $25 \mathrm{~nm}$; de tels filtres avaient jusqu'à présent le défaut d'arrêter aussi une grande partie des protéines et notamment de l'hormone de croissance. Il suffit de les prétraiter par une solution contenant du dextrane, de la sérumalbumine humaine et du polyvinylpyrrolydone à des concentrations appropriées pour, qu'après lavage, l'hormone de croissance se retrouve intégralement dans le filtrat. Les auteurs pensent que ce temps de filtration devrait être ajouté à titre d'étape finale dans le protocole de purification de l'hormone.

J.-C. D.

I. Brown P, Gajdusek DC, Gibbs CJ Jr, Asher DM. Potential epidemic of Creutzfeldt-Jakob disease from human growth hormone therapy. $N$ Engl 7 Med 1985; 313: 728-31.

2. Sauclières G, Allais C. L'hormone de croissance est-elle dangereuse? La Recherche 1985; I6: I392-4.

3. Taylor DM, Dickinson AG, Fraser H, et al. Preparation of growth hormone free from contamination with unconventional slow viruses. Lancet 1985 ii: $\mathbf{2 6 0 - 2}$.

4. Tateishi J, Kitamoto $T$, Hiratani $H$. Creutzfeldt-Jakob disease pathogen in growth hormone preparations is climinatable. Lancet 1985, ii: $1299-300$.

\section{Sonctionnement}

\section{autocrine des}

\section{cellules et cancer}

Le mécanisme de la cancération cellulaire peut être le fonctionnement autocrine d'une cellule qui produit ellemême un facteur de croissance auquel elle est sensible [I]. Un oncogène viral, v-sis, est ainsi l'équivalent du facteur de croissance PDGF (Platelet-Derived Gromth Factor), l'hyperproduction par les cellules infectées d'une telle substance expliquant probablement leur tumorigénicité [2]. De nombreux types tumoraux produisent un facteur de croissance tumoral TGF (Tumor Gromth Factor) [3] qui joue sans doute un rôle déterminant dans la stimulation de la prolifération cellulaire.

Une récente expérience de chercheurs australiens vient de démontrer que 
Thyperexpression par une cellule hématopoiétique d'un facteur de croissance spécifique, un CSF (Colony Stimulating Factor), suffisait à la rendre tumorigène [4]. Une lignée hématopoiétique de souris sensible au "GM-CSF" (Granulocyte-Macrophage Colony Stimulating Factor), et absolument dépendante de lui pour sa prolifération, a été transformée par un rétrovirus recombiné contenant l'ADN complémentaire codant pour ce facteur. Ces cellules prolifereraient alors indépendamment de l apport de CSF exogène et, injectées à une souris syngénique, se sont révélées tumorigènes.

Cet exemple apporte une illustration supplémentaire à la conception maintes fois illustrée dans médecinel sciences : la base moléculaire du cancer est une perte du contrôle des mécanismes physiologiques de régulation de la prolifération cellulaire.

A. $K$.

I. Sporn MB, Roberts AB. Autocrine growth factors and cancer. Nature 1985; 313:745-7.

2. Waterfield MD, Scrace GT, Whittle N, et al. Platelet-derived growth factor is structurally related to the putative transforming protein $\mathbf{p} 28^{\text {sis }}$ of simian sarcoma virus. Nature 1983; 304: 35-9.

3. De Larco JE, Todaro GG. Growth factors from murine sarcoma virus-transformed cells. Proc Natl Acad Sci USA 1978; 75: 400I-5. 4. Lang RA, Metcalf I, Gough NM, et al. Expression of a hemopoietic growth factor cDNA in a factor-dependent cell line results in autonomous growth and tumorigenicity. Cell 1985; 43: 531-42.

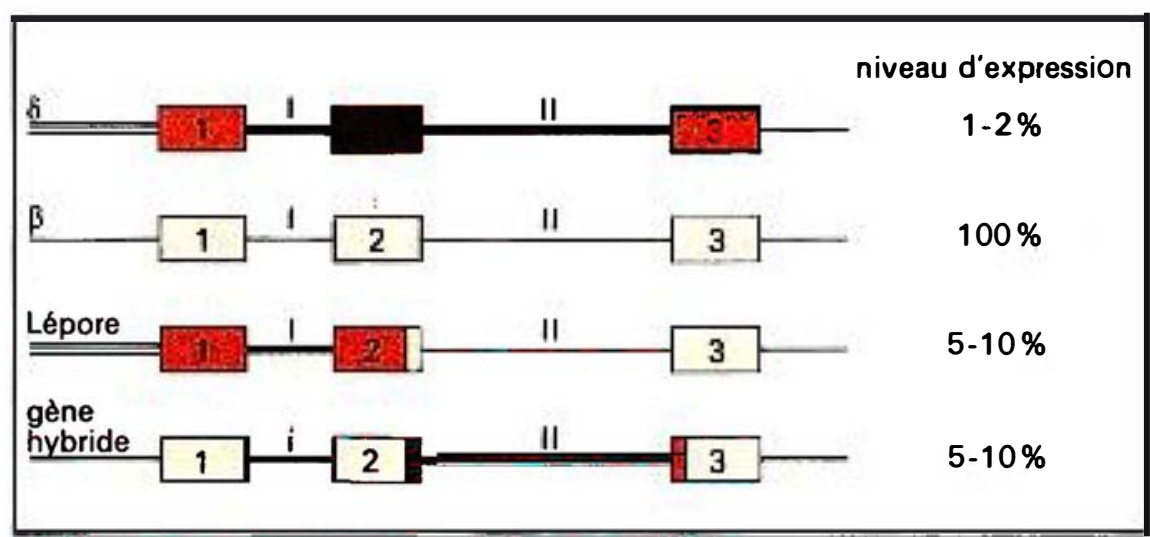

Figure 1. - Niveaux d'expression comparés de gènes $\beta$ et $\delta$ et des gènes hybrides naturels (hémoglobine Lépore) et artificiel. Les exons $d u$ gène $\beta$ sont en rose et ceux du gène $\delta$ en rouge vif. Les introns du gène $\beta$ sont représentés par un trait fin et ceux du gène $\delta$ par un trait épais.

L'existence des introns, ces segments d'ADN qui interrompent en de multiples endroits la séquence codante des gènes, reste uñ grande énigme de la biologie moderne. D'où viennent-ils, que sont-ils, à quoi servent-ils? Autant de questions qui demeurent à ce jour sans réponse. C'est dire l'intérêt de la récente découverte montrant qu'ils peuvent contenir des éléments du contrôle de la transcription des gènes.

On savait déjà, depuis 1983 , qu'après réarrangement des gènes des immunoglobulines au cours de la différenciation des lymphocytes B, un "enhancer ", situé dans l'intron séparant les exons codant pour la partie variable et la partie constante, activait le promoteur du gène [I]. Le gène codant pour l'hormone de croissance est régulé par les glucocorticoïdes, qui en augmentent la transcription; le site majeur de fixation du complexe hormonerécepteur (voir Lexique médecinel sciences $n^{\circ} I$, vol. 2) semble lui aussi situé dans un intron [2]. Les gènes codant pour la chaîne $\beta$ de l'hémoglobine et ses équivalents sont très proches les uns des autres. En particulier le gène $\delta$, codant pour la

$\mathrm{m} / \mathrm{s} n^{\circ} 4$ vol. 2 avril 86 chaîne $\delta$ de l'hémoglobine $A_{2}$ (de formule $\alpha_{2} \delta_{2}$ ) est très peu différent du gène $\beta$ tant au niveau de sa séquence flanquante $5^{\prime}$ (contenant le promoteur et les éléments de régulation amont) que de ses exons. La transcription maximale du gène $\delta$ n'est cependant que de I à $2 \%$ de celle du gène $\beta$. La raison de cette importante différence dans les niveaux d'expression a été analysée par la construction de gènes hybrides (voir figure I). Le remplacement du deuxième intron de $\beta$ par celui de $\delta$ suffit à réduire à 5 -I $0 \%$ de la normale le niveau d'expression du gène $\beta$, alors que le même remplacement au niveau du premier intron est sans effet [3].

En pathologie, il existe un syndrome thalassémique dû a un "crossing over non équationnel " avec production d'un gène hybride comportant les séquences flanquantes $5^{\prime}$, les exons $\mathrm{I}$ et 2 et l'intron I du gène $\delta$, liés à l'intron 2 et à l'exon 3 du gène $\beta$ (figure $I$ ). Ce gène "Lépore" est exprimé au moins 5 fois plus que le gène $\delta$ non remanié. Le deuxième intron des gènes $\delta$ et $\beta$ semble donc contenir des signaux intervenant négativement pour $\delta$ et positivement pour $\beta$ dans le niveau de transcription des gènes. Les exemples semblent donc se multiplier de séquences de contrôle situées dans les introns, ce qui pourrait représenter un moyen d'affiner la régulation de l'expression du génome des organismes supérieurs.

A. $\mathbf{K}$.

I. Banerii J, Olson L, Schaeffner W. A lymphocyte specific cellular enhancer is located downstream joining region in immunoglobulin heavy chain genes. Cell 1985; 33: 729-40.

2. Stater EP, Rabeneau O, Karim M. et al. Glucocorticoïd receptor binding and activation of a heterogenous promotor by dexamethasone by the first intron of the human growth hormone. Mol Cell Biol 1985; 5: 2984-92.

3. Kosche K, Dobkin C, Bank A. DNA sequences regulating human $\beta$ globin gene expression Nucleic Acids Res 1985; 13: 7781-93. 